\subsection{1.-05.11.2011}

Hamburg

Deutschland

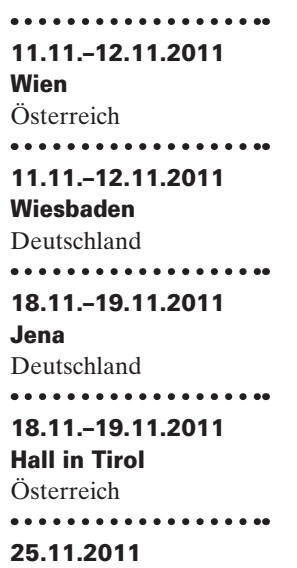

25.11.2011

Augsburg

Deutschland

$25.11-26$.

Krefeld

Deutschland

(...................

25.11.-26.11.2011

Wien

Österreich

-...................

01.12.-03.12.2011

Hamburg

Deutschland

\section{$02.12-03.12 .2011$}

Linz

Österreich

.................

20.01.-21.01.2012

München

Deutschland

\subsection{1.-21.01.2012 \\ Berlin}

Deutschland
Auskunft: $\mathrm{COCS} \mathrm{GmbH}$

Congress Organisation C. Schäfer

Franz-Joseph-Straße 38

80801 München

Tel. +49 89 30710-11, Fax -21

www.cocs.de

\section{Endoskopie Postgraduiertenkurs (ÖGGH)}

1. Endoskopie-Sonografie-Update

\section{Symposium der Arbeitsgemeinschaft Kinderurologie}

der Deutschen Gesellschaft für Kinderchirurgie

Update Gastroenterologie 2011

Endo-Update 2011

186. Tagung der Rheinisch-Westfälischen Gesellschaft für Innere Medizin

\section{Endoskopiekurs für Anfänger (ÖGGH)}

188. Tagung der Vereinigung Nordwestdeutscher Chirurgen

EASIE EMR und ESD (ÖGGH)

\section{Gastro Forum München}

6. Jahrestagung der Gesellschaft für Gastroenterologie und

Hepatologie in Berlin und Brandenburg e.V. (GGHBB) zusammen mit dem

3. Symposium für Endoskopie- und Assistenzpersonal

\section{Onkologie-Update-Seminar}

Auskunft: azmedinfo@media.co.at www.oeggh.at

Auskunft: kathrin.schoppa@ed-update.com www.endo-sono-update.com

Auskunft: ag-kinderurologie@dgkch.de www.kinderchirurgie.uniklinikum-jena.de

Auskunft: azmedinfo@media.co.at

Auskunft: Sekretariat III, Medizinische Klinik,

Klinikum Augsburg

Frau Konzet

gudrun.konzet@klinikum-augsburg.de

www.klinikum-augsburg.de

Auskunft: www.rwgim.de

Auskunft: azmedinfo@media.co.at

www.oeggh.at

Auskunft: MCN Medizinische Congressorganisation Nürnberg AG

Frau Reimer

Tel. +49 911 39316-40, Fax -66

reimer@mcn-nuernberg.de

www.mon-nuernberg.de

Auskunft: azmedinfo@media.co.at

www.oeggh.at

Auskunft: $\mathrm{COCS} \mathrm{GmbH}$

Congress Organisation C. Schäfer

Tel. +49 89 890677-0, Fax -77

Frau Reber

sandra.reber@cocs.de

www.cocs.de

Auskunft: Charité, Virchow-Klinikum Berlin,

Medizinische Klinik mit Schwerpunkt Hepatologie

und Gastroenterologie

Tel. +49 30 326413-02, Fax -00

www.gghbb.de

Auskunft: www.onko-update.com

Deutschland

\section{KARGER}

Fax +497614520714

Information@Karger.de

www.karger.com (c) 2011 S. Karger GmbH, Freiburg 


\subsection{2.-04.02.2012}

Düsseldorf

Deutschland

[...............

\subsection{2.-04.02.2012}

Wiesbaden

Deutschland

(1..................

22.02.-25.02.2012

Berlin

Deutschland

.................

25.02.2012

Ludwigshafen

Deutschland

\subsection{3.-04.03.2012}

Nürnberg

Deutschland

-

22.03.-24.03.2012

München

Deutschland

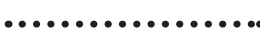

22.03.-24.03.2012

St. Gallen

Schweiz

\section{...................}

24.04.-27.04.2012

Berlin

Deutschland

\section{[................}

27.04.-28.04.2012

Essen

Deutschland

..................

10.05.-12.05.2012

Jena

Deutschland

................

07.06.-08.06.2012

Hannover

Deutschland

\subsection{7.-07.07.2012}

Baden-Baden

Deutschland

\section{..................}

06.09.-08.09.2012

Bologna

Italien

[.................

12.09.-15.09.2012

Berlin

Deutschland
14. Internationales Endoskopie Symposium Düsseldorf

7. Onkologie-Update-Seminar

30. Deutscher Krebskongress

VII. "LUKS" - Ludwigshafener und Kölner Stentworkshop

Gemeinsamer Bundeskongress Chirurgie 2012

42. Kongress der Deutschen Gesellschaft für Endoskopie und Bildgebende Verfahren e.V.

1st St. Gallen EORTC Gastrointestinal Cancer Conference (SG-GICC 2012)

129. Kongress der Deutschen Gesellschaft für Chirurgie (DGCH)

\section{Tagung der Gesellschaft für Gastroenterologie in} Nordrhein-Westfalen e.V.

21. Kongress der Mitteldeutschen Gesellschaft für Gastroenterologie

D-Notes 2012 - Natural Orifice Translumenal Endoscopic Surgery (Arbeitsgruppe Deutschland)

49. Kongress der Südwestdeutschen Gesellschaft für Innere Medizin

Joint International Neurogastroenterology and Motility Meeting (NGM 2012)

9. Kongress der Deutschen Gesellschaft für Palliativmedizin
Auskunft: COCS GmbH Congress Organisation

C. Schäfer

Frau Reber

sandra.reber@cocs.de

www.endo-duesseldorf.com

Auskunft: www.onko-update.com

Auskunft: info@dkk2012.de

www.dkk2012.de

Auskunft: COCS GmbH Congress Organisation

C. Schäfer

Frau Gautsch

eva.gautsch@cocs.de

www.cocs.de

Auskunft: MCN Medizinische Congressorganisation Nürnberg AG

Tel. +49 911 39316-39, Fax -20

bnc@mcnag.info

www.mon-nuernberg.de

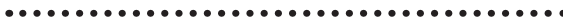

Auskunft: COCS GmbH Congress Organisation

C. Schäfer

Frau Wiederkrantz

Tel. +49 89 890677-0, Fax -77

martina.wiederkrantz@cocs.de

www.dge-bv.de

Auskunft: St. Gallen Oncology Conferences

c/o Tumor and Breast Center ZeTuP

info@oncoconferences.ch

www.oncoconferences.ch

Auskunft:

Nürnberg AG

Frau Veith

veith@mcn-nuernberg.de

www.chirurgie2012.de

Auskunft: gastronrw@uk-essen.de

www.gastronrw.de

Auskunft: www.mgfg.de

Auskunft: Diakoniekrankenhaus

Henriettenstiftung gGmbH

Frau Krupp

gabriele.krupp@ddh-gruppe.de

www.d-notes2012.de

Auskunft: MedCongress GmbH

Frau Schäfer

gs@medcongress.de

www.swgim.de

Auskunft: CPO HANSER SERVICE GmbH

ngm2012@cpo-hanser.de

www.ngm2012.org

Auskunft: INTERPLAN Congress,

Meeting \& Event Management AG

dgp2012@interplan.de

www.dgp2012.de 


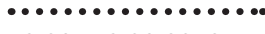

12.09.-16.09.2012

Hamburg

Deutschland

108. Jahrestagung der Deutschen Gesellschaft für Kinder- und Jugendmedizin e.V.

\subsection{9.-29.09.2012}

Karlsruhe

Deutschland

- $\ldots \ldots \ldots \ldots \ldots \cdots \cdots$

19.10.-23.10.2012

Stuttgart

Deutschland

28.06. 29.06 .2013

Hannover

Deutschland

Jahrestagung der Deutschen Gesellschaft für Hämatologie und Onkologie e.V. (DGHO 2012)
Auskunft: INTERPLAN Congress,

Meeting \& Event Management AG

Herr Hausfeld

Tel. +49 30 9210466-53, Fax -99

t.hausfeld@interplan.de

www.dgkj2012.de

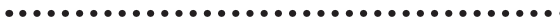

Auskunft: MCN Medizinische Congressorganisation Nürnberg AG

mcn@mcn-nuernberg.de

www.dgt-online.de

Auskunft:kongress2012@dgho-service.de www.haematologie-onkologie-2012.de

www haematologie-onkologie-2012,

Auskunft: Diakoniekrankenhaus

Henriettenstiftung gGmbH

www.complications-in-endoscopy.com 